\title{
4th Colloquium Interdisciplinary in Instrumentation
}

This special issue of The European Physical Journal is based on a choice of papers selected by the scientific committee of the Fourth Conférence Interdisciplinaire en Instrumentation (C2I'2007) which was held at Nancy in October 2007.

This conference was created in 1998 in the École Normale Supérieure de Cachan, where the first event was organized in November 1998. For this initial experience, our aim was to propose to scientists coming from different disciplines to present their works in the interdisciplinary area of instrumentation. People came mostly from French laboratories and industries; however, papers from ten other countries were presented, among approximately a total of 120 papers of that conference. Numerous scientific fields were covered, from fundamental sciences to applications in physics, biology, chemistry, and so on. Second and third conferences were organized in Paris in 2001 and 2004 respectively, with approximately the same level of success, with regard to both participation and quality of papers. For all these events the Eur. Phys. J. Appl. Phys. was a partner and published a selection of papers from those presented in the C2I.

This special issue is composed of six papers, elected from a selection of thematic made by the scientific committee, covering a wide panel of areas, with very interesting applications in medical fields, music, ultrasonic imaging, laboratory on chips and nano-objects imagin. Selection of these fields and papers was difficult, because of the high quality of the papers presented at C2I'2007, and also because our aim was to point out the diversity in this field of instrumentation.

Some of the papers are devoted to the conception and the characterization of specific sensors: defining optimal excitation of an ultrasonic transducer assuring the functions of automatic tuning and impedance matching, development of a gas sensor based on a surface acoustic wave device, development of a sensor for detecting nanoparticles. Two papers are concerned with the use of sensors: the observation of magneto ionosphere responses to $P$ wave emissions during an earth quake, bimodal spectroscopy for discriminating mouse skin types. The last paper presents both the conception and the use of a sensor for vibration analysis.

Issue after issue, we have seen through the works presented by researchers and engineers that performing reliable and complex instrumentation leads to improved understanding of the physical (or chemical, biological... ) phenomenon. It also increases the synergy between disciplines that helps to achieve more sophisticated progresses in instrumentation design and performance. We hope that the reader will find in this journal a good illustration of these concepts, through this short selection of papers. Expecting that our range of knowledge should ever widening, we are currently preparing the next edition of the C2I, which will be held in Le Mans in January 2010. 